\title{
3D Morphology of Magnetic Bubbles in Layered Ferromagnetic Materials
}

\author{
Rich Moraski ${ }^{1}$ and Benjamin McMorran ${ }^{2}$ \\ ${ }^{1}$ University of Oregon, Eugene, Oregon, United States, ${ }^{2}$ University of Oregon, United States
}

In the late 1960's, engineers at Bell Labs observed magnetic domains that were arranged in cylindrical shapes they

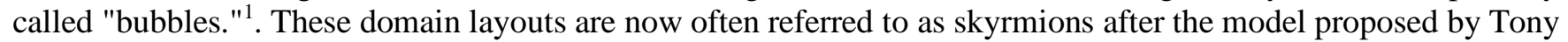
Skyrme in 1961 to describe nucleons ${ }^{2}$. Skyrmions are topological solitons, or self-reinforcing fields that behave like particles $^{3}$. They can be caused by non-centrosymmetric or topologically defective crystal lattices. In the case of layered materials that lack inversion symmetry (e.g., alternating thin films of iron and gadolinium), an additional source of skyrmions is the antisymmetric exchange interaction, or $\mathrm{DMI}^{4,5}$ (named for Igor Dzyaloshinsky and Toru Moriya who first wrote about them in the late 1950's), that occurs at the interfaces of the layers ${ }^{6}$.

An example of magnetic skrymions observed with a transmission electron microscope operating in Lorentz mode (L-TEM) is shown in Figure 1. The arrows in this image show the direction of the average magnetization through the bulk of the sample in the area imaged. Similar images have been captured using scanning electron microscopes (SEMs), showing the in-plane magnetization at the surface of the sample. Using a unique tool originally built at NIST and now operated at UO, SEMPA, or scanning electron microscope with polarization analysis, the magnetization at sample surfaces has also been observed to extend out of the plane of the sample ${ }^{7}$.

SEMPA quantitatively maps the vector components in 3 dimensions of the magnetization of sample surfaces. Spin polarized secondary electrons are directed toward a gold target and Mott scattered to a direction-sensitive sensor. L-TEM measures a magnetic flux density through a sample, deflecting the electron beam via the Lorentz force, and maps a projection of the vector components of that field transverse to the beam, predominantly in the plane of the sample. SEMPA uses a lower accelerating voltage, typically $25 \mathrm{kV}$, and measures a signal from only the first few angstroms of the sample surface instead of passing through the entire bulk.

The McMorran group is collaborating with Sergio Montoya and Eric Fullerton of the Center for Magnetic Recording Research (CMRR), University of California, San Diego, on characterizing amorphous Fe/Gd thin films using L$\mathrm{TEM}^{8}$. Dr. Montoya is producing room temperature, external field-free stable skyrmions in various layered thin films of iron, gadolinium, platinum, and iridium where perpendicular magnetic anisotropy rather than DMI is the primary contributor to skyrmion nucleation. Early analysis of an 80 layer sample is presented in Figure 2.

This research combines results from SEMPA along with L-TEM to provide measurements, analysis, and visualization of samples with varying layer thicknesses and compositions, enabling direct comparison of bulk and surface fields, to understand how different anisotropies affect skyrmion phases. The ultimate goal is to identify parameters required to engineer materials with specific domain chirality.

Magnetic bubbles require significantly less power to manipulate than traditional electronics, opening a number of potential uses in spintronics, computing, and other fields ${ }^{9}$. Periodic morphologies such as hopfions also have additional degrees of freedom that might be useful for encoding information. 


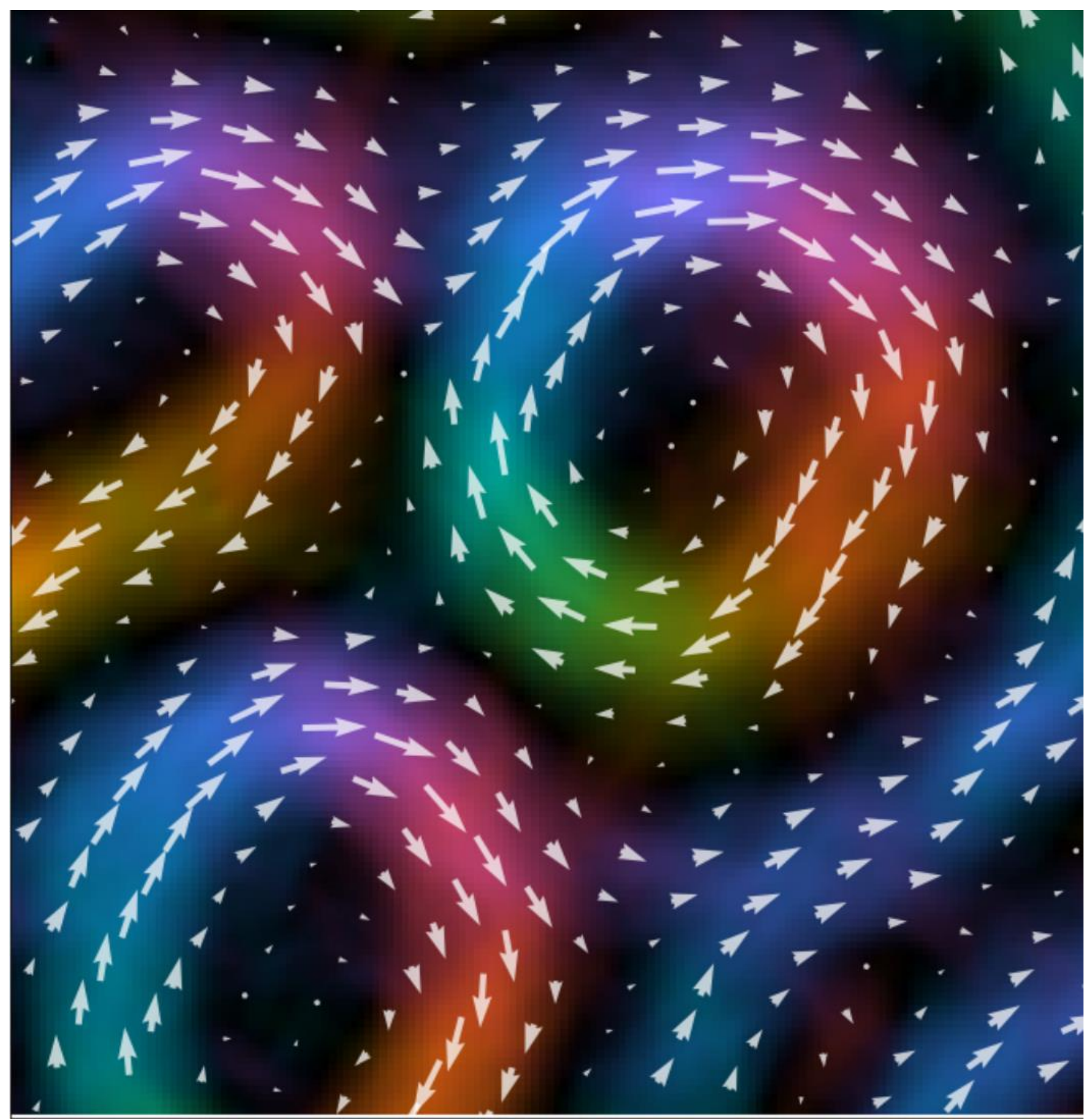

Figure 1. Skyrmions imaged using L-TEM for an 80 layer Fe/Gd sample from CMRR 


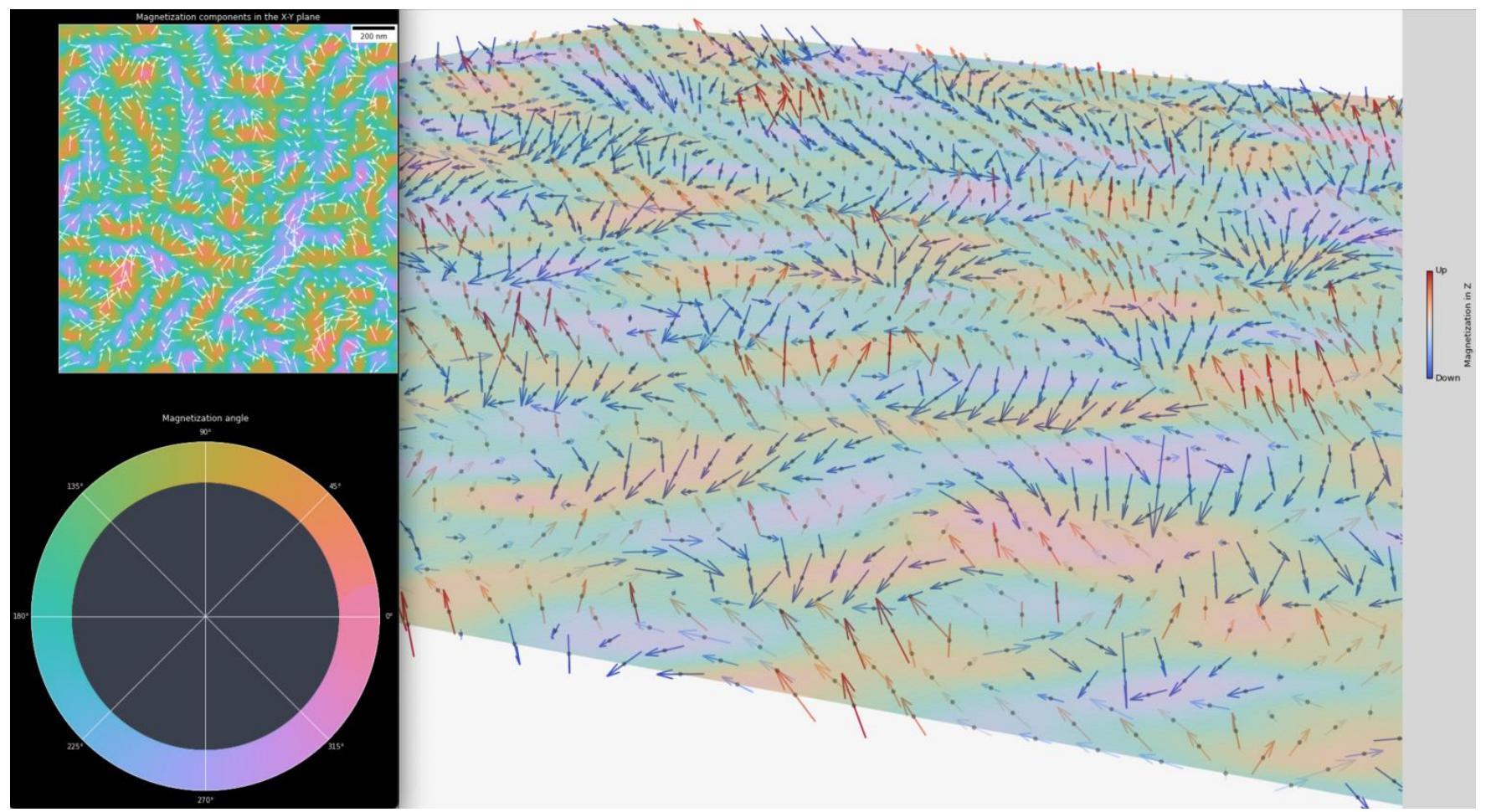

Figure 2. Another 80 layer Fe/Gd heterostructure was imaged in 3 dimensions with SEMPA revealing Néel caps around the magnetic domains suggesting a rich morphology

\section{References}

1. Andrew H. Bobeck and H. E. D. Scovil. "Magnetic Bubbles". In: Scientific American 224.6 (1971), pp. $78-91$. issn: 00368733, 19467087. url: http://www.jstor.org/stable/24922754.

2. T. H. R. Skyrme. "A Unified Field Theory of Mesons and Baryons". In: Nuclear Physics 31 (1961), pp. 556559.

3. P. G. Drazin and R. S. Johnson. Solitons: an introduction. Cambridge University Press, 1989.

4. I. Dzyaloshinsky. "A Thermodynamic Theory of "Weak" Ferromagnetism of Antiferromagnetics". In: Journal of Physics and Chemistry of Solids 4 (1958), pp. 241-255.

5. T. Moriya. "Anisotropic Superexchange Interaction and Weak Ferromagnetism". In: Physical Review 120.1 (1960), pp. 91-98.

6. A. Crépieux and C. Lacroix. "Dzyaloshinsky-Moriya interactions induced by symmetry breaking at a surface". In: Journal of Magnetism and Magnetic Materials 182 (1998), pp. 341-349.

7. J. J. Chess. "Mapping Topological Magnetization and Magnetic Skyrmions". PhD thesis. Univer- sity of Oregon, 2017.

8. J. Chess et al. "Observation of Skyrmions at Room-temperature in Amorphous Fe/Gd Films". In: Microscopy and Microanalysis 21 (2015), pp. 1649-1650.

9. Spintronics for Low-Power Computing. Design, Automation, Test in Europe Conference, and Exhibition (DATE). 2014 